\title{
Approaching Two-dimensional Polymers with Macroscopic Sizes
}

\author{
Payam Payamyars, Junji Sakamoto, and A. Dieter Schlüter
}

§SCS-DSM Award for best poster presentation

\begin{abstract}
We describe the challenges involved with extending the limited lateral size of two-dimensional polymers (2DPs). An amphiphilic monomer with three-fold symmetry is chosen to form an ideally tessellated monolayer at the air/water interface. Anthracene [4+4] photo-dimerization is chosen as the growth reaction. Formation of covalent net-points upon anthracene dimerization has an influence on the mechanical coherence of the resulting sheets which could be investigated qualitatively and quantitatively by means of AFM nano-indentation.
\end{abstract}

Keywords: Air/water interface · Exfoliation · Long-range order · Monolayers · Photo-dimerization

\section{Introduction}

The common definition of polymers, which was introduced by Staudinger in the 1920 s, refers to giant molecules, called in German 'Makromoleküle', in which repeat units are covalently bonded to one another along their topologically linear binding sites. ${ }^{[1]}$ Needless to say, these macromolecules in the meantime have 'conquered' the world by providing countless materials for basically all aspects of our lives. In such polymers, entanglements play a key role in regard to properties; they provide elasticity and strength. The modern understanding of materials as a whole and polymers in particular raised awareness about the importance of surfaces besides the entanglements in providing novel properties. This notion laid the first steps towards a more systematic analysis of the dimensionality of materials. ${ }^{[2]}$ The advent of graphene, a sheet-like object, in the first decade of the $21^{\text {st }}$ century made the importance of this realization even more vivid. ${ }^{[3]}$ Classification of materials as 1D, 2D and 3D triggered a new insight into the characteristics of each dimension and their impact on materials

\footnotetext{
${ }^{\star}$ Correspondence: Prof. A. D. Schlüter Institut für Polymere

ETH Zürich

Wolfgang-Pauli-Str. 10

$\mathrm{CH}-8093$ Zürich

Tel.: +41446336380

E-Mail: dieter.schlueter@mat.ethz.ch
}

properties. ${ }^{[2]}$ Applying this approach to what Staudinger had introduced, classifies his 'Makromoleküle' as one-dimensional polymers (1DPs). ${ }^{[4]}$

Polymer chemistry has made great progress in the understanding of structural issues related to one-dimensional polymers (1DPs). Control of molecular weight, molecular weight distribution, stereochemistry and nature of end groups is nowadays almost a matter of routine. However in terms of dimensionality, the developments are way behind what nature can achieve. There is no rational synthesis of a regular sheet such as graphene, ${ }^{[5]}$ a two-dimensional poymer (2DP), and there is no rational synthesis of diamond, a three-dimensional polymer (3DP). Thus, the concepts which so far were so successfully developed for 1DPs cannot yet be easily applied to polymers with higher dimensionalities (Fig. 1a,b).

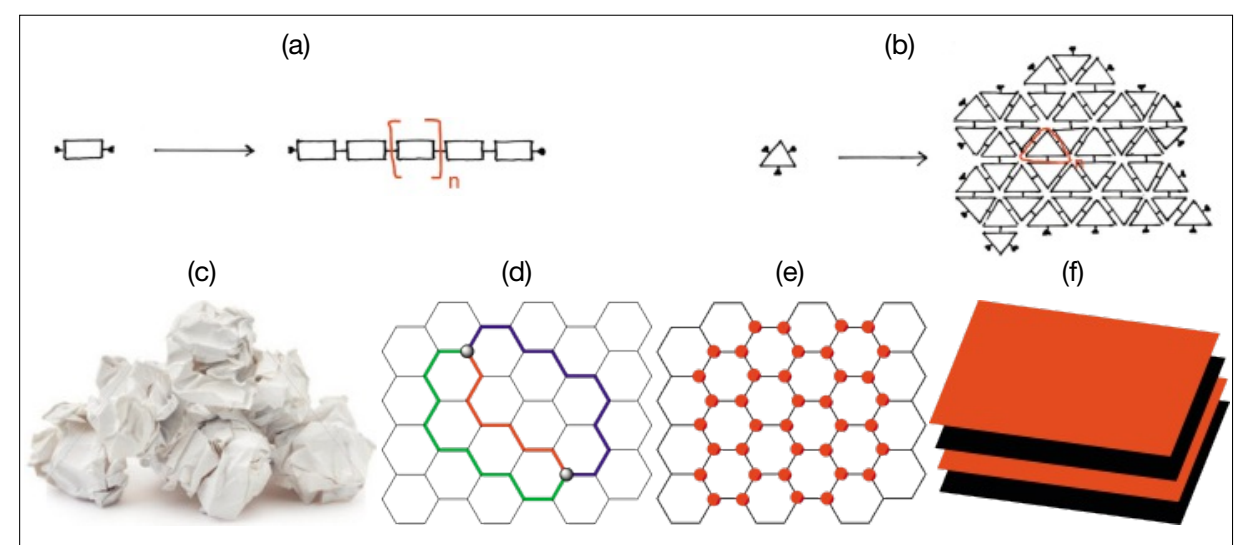

Fig. 1. (a) 1D polymerization, (b) 2D polymerization, (c) crumplings instead of entanglements in 2DPs, (d) multiple lateral cross-talk channels in 2DPs, (e) 2DPs as scaffolds for ordered functionalization and for 3D constructions (f). covalent bonds of a double-stranded polymer to a larger lateral dimension made by the Schlüter's group came to a new realization of dimensionality in polymers. In 2009, they defined a monolayer made up of covalently bonded, topologically planar repeat unit with long-range molecular order as a 2DP. ${ }^{[4]}$ In this context, graphene could be thought of a naturally occurring 2DP in which the $\mathrm{sp}^{2}$-hybridized carbon atom serves as the repeat unit.

Unlike the linear polymers, 2DPs have no chance for entanglement and merely crumple upon lateral compression (Fig. 1c). They have multiple cross talk channels which are, in principle, ideal, e.g. for electrically conducting 2DPs (Fig. 1d). Rational synthesis provides sheets where not only the nature of pendant functional groups can be deliberately chosen but also these groups' exact positioning across both
The attempts towards extending the 
sides of the sheet can be controlled by the monomer design (Fig. 1e). This fascinating opportunity for structure control on a molecular level is the element that makes rational, organic chemistry-style synthesis different from thermolytic procedures. ${ }^{[5]}$ Finally, 2DPs can be used for the systematic construction of 3D matter by ordered stacking (Fig. 1f).

Attempts towards the creation of 2DPs were triggered by the synthesis of monomers with at least three binding sites on their periphery. ${ }^{[6]}$ It was important to have monomers that pack in 2D tessellated ensembles with their binding sites in close vicinity of each other to render lateral polymerization possible. Two methods are presently being pursued for this purpose. One is the crystallization of the monomers that results in stacked ordered lamellae of the tessellated assembly, similar to that of graphite, which need to be exfoliated after polymerization. The other technique is the use of the air/water interface to form an ordered 2D array of an amphiphilic monomer on the water. The former technique has the combined advantages of potentially providing highly ordered sheets with a more facile access to structural analysis after polymerization. But the downside is the fact that it produces sheets with lateral sizes limited to that of the crystal; also the difficulties associated with exfoliation of the polymerized sheets need to be considered. The latter method, however, can provide much larger sheets but bears the disadvantage of having less structural perfection along with the challenges involved with characterization of the final monolayer.

\section{Results}

For the first time, rational synthesis of a 2DP was realized upon crystallization of monomer 1 which resulted in a lamellar structure shown in Fig. 2c. ${ }^{[7]}$ The close proximity of each anthracene moiety from one monomer to ethynylene of its neighboring monomer made a [4+2] photo-induced cycloaddition possible as the growth strategy. ${ }^{[7]}$ The exfoliation of the irradiated crystals resulted in sheets with the thickness that is expected from a monolayer.

While we consider this an important finding, it has the limitation that the sizes of the created 2DPs are intrinsically limited by the crystal size. Typical crystal sizes range between 1-100 $\mu \mathrm{m}$ which prevents applications that require $\mathrm{mm}$ - or even $\mathrm{cm}$ sized sheets. ${ }^{[7]}$ It was therefore of importance to overcome this limitation by embarking on the use of the air/water interface as a substrate for inducing order among the monomers prior to polymerization. ${ }^{[9]}$ We however maintained photochemistry as the tool to connect monomers with one

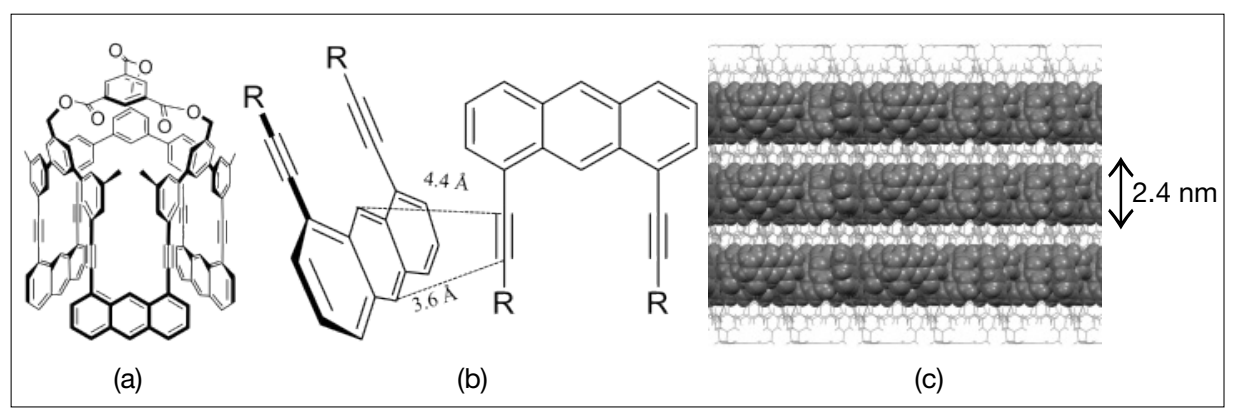

Fig, 2. (a) Monomer 1 used for 2DP synthesis by means of [4+2] photoinduced cycloaddition, (b) distances between the anthracene and ethynylenes moieties of neighboring monomers allowing for photochemically triggered cycloaddition, (c) crystal structure of monomer 1 showing the layered structure which is prerequisite for lateral polymerization to 2DP. The monomers are oriented alternatingly up and down. The photochemically sensitive anthracene and ethynylene moieties are represented as space filling models.

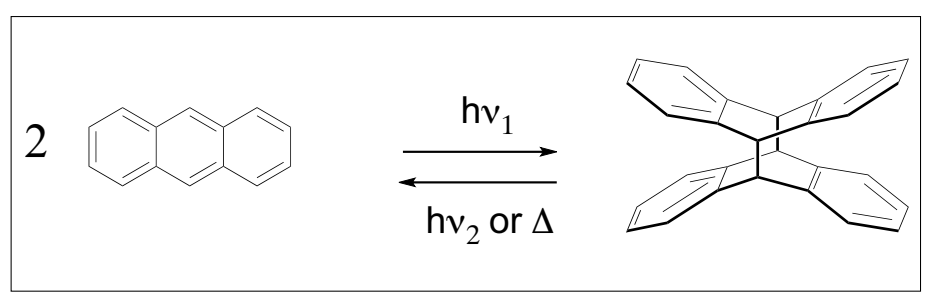

Scheme 1. The reversible anthracene dimerization which is the key reaction in the present polymerization of monomer $\mathbf{2}$, comprised of three anthracene units. ${ }^{[8]}$

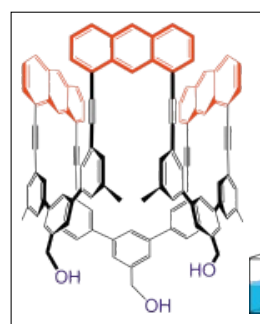

(a)

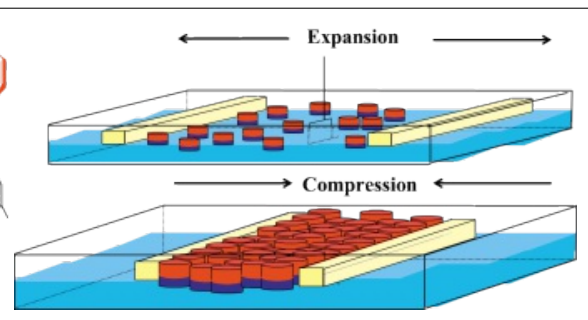

(b)

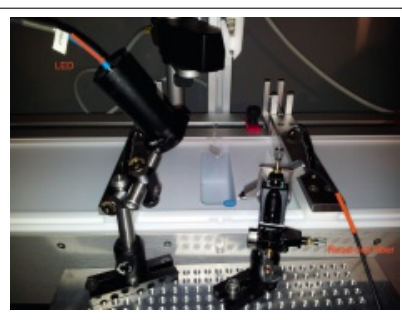

(c)

Fig. 3. (a) Chemical structure of the amphiphilic monomer 2 used in the present study, (b) schematic representation of the experiment on a Langmuir trough, ideally resulting in a tessellated layer of monomers at the air/water interface ready to be laterally polymerized by light irradiation and (c) experimental set-up with an LED (left) and an emission detector mounted on a Langmuir trough (right). Note that the blue mark is artificially introduced to indicate that only a relatively small area of the compressed film actually gets irradiated.

another due to its invaluable advantage of avoiding mass transport. ${ }^{[10]}$ Macrocycle 2, which is a congener of $\mathbf{1}$, was selected for this purpose. ${ }^{[6 b]}$ Its amphiphilic nature was expected to render a preferred arrangement of the molecule possible at the interface with the hydrophilic base of the cycle facing the water subphase and the hydrophobic part sticking out. We expect that the anthracenes would be at the same height away from the water with a face-to-face orientation which would make a [4+4] cycloaddition of the adjacent monomers possible (Scheme 1).

The anthracene dimerization reaction path is known to have an excimer as intermediate, with a fluorophore reactant and a quenched dimer as the product. ${ }^{[8]}$ We therefore aimed at in situ fluorescence emission spectroscopy of the layer at the interface as a way to prove polymerization (Fig. 3c).

Given that anthracene dimerization is a reversible reaction, the growth reaction at the interface would, in principle, result in a degradable polymer that has a potential for producing well-defined patterns on it by means of deep UV lithography. This polymer could be used as a membrane with well-defined pore sizes. The amphiphilic nature of the constituent monomer makes the final sheet consist of different faces. One face is hydrophilic with the hydroxyl groups sticking out and the other one is hydrophobic with the anthracene dimers on it. Such a difference in the nature of the faces are desirable for, e.g. biological applications.

Due to the potentially adverse effect of oxygen on the formation of netpoints (anthracene dimers) in the resulting sheets, it is desirable to study the effect of oxygen on the polymerization. Oxidation of the anthracenes ${ }^{[11]}$ would result in anthracene endoperoxide or anthraquinones that are both detrimental for bond percolation within 
the sheet and therefore undesirable for the aimed structural perfection. Qualitative analysis of the formed film by microscopy techniques along with quantitative spectroscopic analysis like the highly sensitive tip-enhanced Raman spectroscopy ${ }^{[12]}$ could provide insights into the oxidation path of the reaction.

Higher content of the anthracene dimerization should bring about more pronounced mechanical properties like elastic modulus and strength. In fact, this could be investigated by AFM nano-indentation experiments ${ }^{[13]}$ which are suitable to study these properties while the film is spanned over grids with circular holes (Fig. 4). The result of these investigations provide Young's moduli in the GPa range which compare well with reference sheets such as graphene if one takes the different netpoint density and sheet thicknesses into consideration (in collaboration with the group of Prof. A. Stemmer, ETH Zürich, Rüschlikon, Switzerland). ${ }^{[9]}$

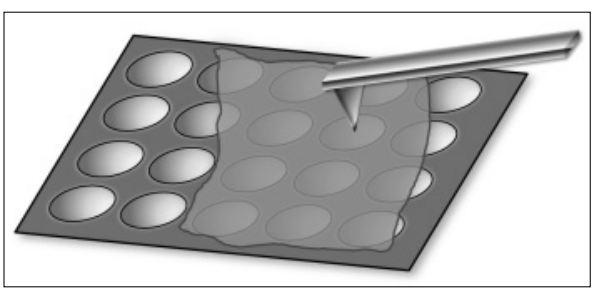

Fig. 4. Schematic representation of nanoindentation, a method to determine Young's modulus, pre-tension and fracture force of sheet-like objects (such as 2DPs).

\section{Conclusion}

Creation of covalently bonded sheets with long-range internal order is possible through the rational synthesis of monomers with at least three binding sites that can form a tessellated ensemble ready to be laterally polymerized. The polymerization reaction can take place either in a crystal or at the air/water interface. Present work is aimed at showing that monomer $\mathbf{2}$ can be converted into a covalent monolayer with $\mathrm{cm}^{2}$ scale lateral size and attractive mechanical stability. First evidence for internal order is based on in situ fluorescence spectroscopy. It should be noted, however, that the final proof of molecular structure will require substantial further research effort and will be sooner or later confronted with the fact that monolayers at interfaces of whatever nature consist of domains. Thus, future research will also concentrate on domain size and its control. 2DPs offer a new class of materials that could be investigated only when the proper analytical techniques for probing the monolayers are well-established.

Received: March 11, 2013

[1] a) H. Staudinger, Ber. Dtsch. Chem. Ges. 1920, 53, 1073; b) H. Staudinger, J. Fritschi, Helv. Chim. Acta 1922, 5, 785. c) H. F. Mark, Naturwiss. 1980, 67, 477; d) H. Ringsdorf, Angew. Chem., Int. Ed. 2004, 43, 1064.

[2] R. Mas-Ballesté, C. Gómez-Navarro, J. GómezHerrero, F. Zamora, Nanoscale, 2011, 3, 20.

[3] K. S. Novoselov, A. K. Geim, S. V. Morozov, D. Jiang, Y. Zhang, S. V. Dubonos, I. V. Grigorieva, A. A. Firsov, Science, 2004, 306, 666
[4] J. Sakamoto, J. van Heijst, O. Lukin, A. D. Schlüter Angew. Chem. Int. Ed. 2009, 48, 1030.

[5] For thermolytic synthesis of graphene see: a) J. Cai, P. Ruffieux, R. Jaafar, M. Bieri, T. Braun, S. Blankenburg, M. Muoth, A. P. Seitsonen, M. Saleh, X. Feng, K. Müllen, R. Fasel, Nature, 2010, 466, 470; b) M. Bieri, M. Treier, J. Cai, K. Ait-Mansour, P. Ruffieux, O. Groning, P. Groning, M. Kastler, R. Rieger, X. Feng, K. Müllen, R. Fasel, Chem. Commun. 2009, 45, 6919.

[6] a) C. Münzenberg, A. Rossi, K. Feldman, R. Fiolka, A. Stemmer, K. Kita-Tokarczyk, W. Meier, J. Sakamoto, O. Lukin, A. D. Schlüter, Chem. Eur. J. 2008, 14, 10797; b) P. Kissel, J. v. Heijst, R. Enning, A. Stemmer, A. D. Schlüter, J. Sakamoto, Org. Lett. 2010, 12, 2778; c) K. Patrick, A. D. Schlüter, J. Sakamoto, Chem. Eur. J. 2009, 15, 8955; d) T. Bauer, A. D. Schlüter, J. Sakamoto, Synlett 2010, 6, 877.

[7] P. Kissel, R. Erni, W. B. Schweizer, M. D. Rossell, B. T. King, T. Bauer, S. Götzinger, A. D. Schlüter, J. Sakamoto, Nature Chem. 2012, 4, 287.

[8] a) H. Bouas-Laurent, A. Castellan, J. P. Desvergne, R. Lapouyade, Chem. Soc. Rev. 2000, 29, 43; b) H. Bouas-Laurent, A. Castellan, J. P. Desvergne, R. Lapouyade, Chem. Soc. Rev. 2001, 30, 248; c) H.-D. Becker, Chem. Rev. 1993, 93,145 .

[9] P. Payamyar, K. Kaja, A. Stemmer, A. Renn, A. Schütz, C. Johnson, D. J. Murray, B. T. King, L.-T. Lee, Z. Zheng, J. Sakamoto, A. D. Schlüter, 2013, to be submitted.

[10] T. Bauer, Z. Zheng, A. Renn, R. Enning, A. Stemmer, J. Sakamoto, A. D. Schlüter, Angew. Chem. Int. Ed. 2011, 50, 7879.

[11] a) W. Fudickar, T. Linker, Langmuir, 2010, 26,6, 4421; b) W. Fudickar, T. Linker, Chem. Eur. J. 2006, 12, 9276.

[12] L. Opilik, T. Bauer, T. Schmid, J. Stadler, R. Zenobi, Phys. Chem. Chem. Phys. 2011, 13, 9978.

[13] C. Lee, X. Wei, J.W. Kysar, J. Hone, Science 2008, 321, 385. 